\title{
GNSS Shadow Matching: Improving Urban Positioning Accuracy Using a 3D City Model with Optimized Visibility Scoring Scheme
}

\author{
LEI WANG, PAUL D. GROVES, and MAREK K. ZIEBART \\ University College London, London, United Kingdom \\ Received October 2012; Revised March 2013
}

\begin{abstract}
Global navigation satellite system (GNSS) positioning is widely used in land vehicle and pedestrian navigation systems. Nevertheless, in urban canyons GNSS remains inaccurate due to building blockages and reflections, especially in the cross-street direction. Shadow matching is a new technique, recently proposed for improving the cross-street positioning accuracy using a 3D model of the nearby buildings. This paper presents a number of advances in the shadow-matching algorithm. First, a positioning algorithm has been developed, interpolating between the top-scoring candidate positions. Furthermore, a new scoring scheme has been developed that accounts for signal diffraction and reflection. Finally, the efficiency of the process used to generate the grid of building boundaries used for predicting satellite visibility has been improved. Real-world GNSS data has been collected at 22 different locations in central London to provide the first comprehensive and statistical performance analysis of shadow matching. Copyright (C) 2013 Institute of Navigation.
\end{abstract}

\section{INTRODUCTION}

The poor performance of global navigation satellite systems (GNSS) user equipment in urban canyons is a well-known problem in terms of both accuracy and solution availability [1-3]. In contrast, a great number of day-to-day navigation requests are made in urban areas by city residents. Advanced intelligent transportation systems, for example, rely on positioning systems for their ability to direct individual cars in order to maximize traffic flow and prioritize emergency vehicles [4]. Vehicle lane detection in lane guidance systems, location-based advertising, augmented-reality applications, and step-by-step guidance for visually impaired and tourists all require sufficient positioning accuracy to perform their functions [5-8]. However, the availability and accuracy of GNSS in urban areas limits the use of these applications [3].

The problem of GNSS performance in urban canyons arises when the direct line-of-sight (LOS) signals from many of the satellites are blocked. The blockage is likely to happen where there are tall buildings or narrow streets. The buildings effectively cast GNSS shadows over the adjacent terrain. Without direct signals from four or more satellites,

Navigation: Journal of The Institute of Navigation

Vol. 60, No. 3, Fall 2013

Printed in the U.S.A. an accurate position solution cannot be determined. Sometimes, a degraded position solution may be obtained by making use of signals that can only be received by reflection off a building; these are known as non-line-of-sight (NLOS) signals [9, 10].

As well as affecting the number of available GNSS signals, an urban canyon also affects the geometry of satellites, which causes lower accuracy in the crossstreet direction. This is because signals with lines of sight going across the street are much more likely to be blocked by buildings than signals with lines of sight going along the street. As a result, the signal geometry, and hence the positioning accuracy, will be much better along the direction of the street than across the street [2]. Figure 1 illustrates this.

For improving navigation performance in highly built-up areas, a variety of navigation sensors have been used to enhance or augment GNSS. Road vehicles typically combine GNSS with odometers, and map-matching algorithms, while pedestrian navigation users may combine GNSS with cell phone signals, Wi-Fi, and/or dead reckoning using inertial sensors, magnetic compass and barometric altimeter $[11,12]$. However, these approaches improve the continuity and robustness of the position solution, but not the cross-street accuracy.

As 3D building models are becoming more accurate and widely available [13, 14], they are increasingly treated as a new data source for urban 


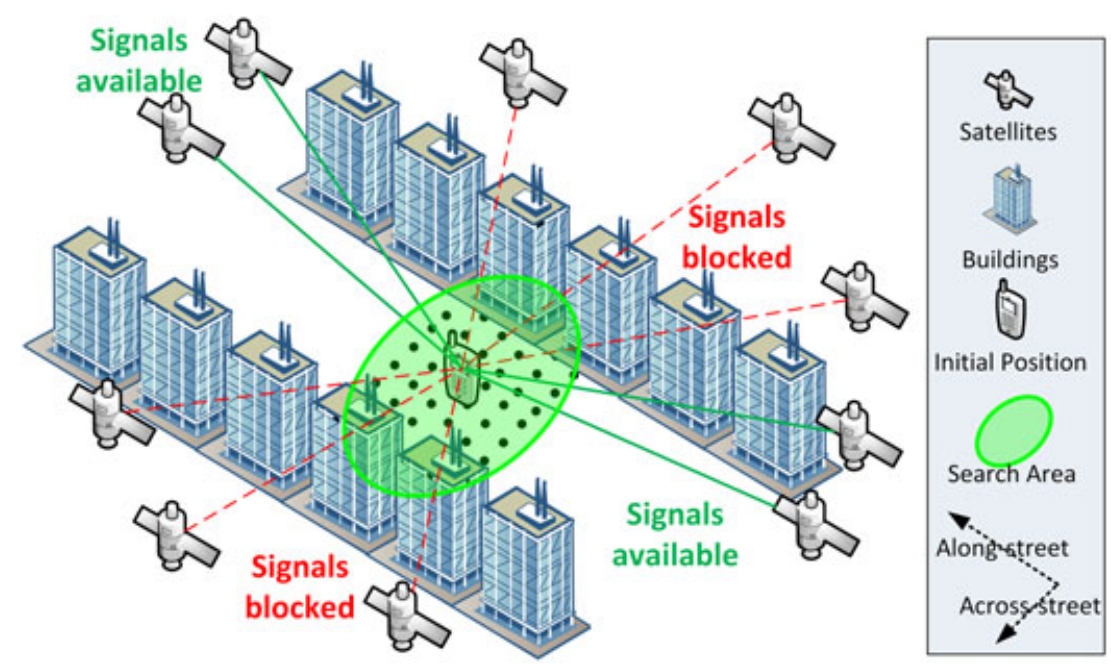

Fig. 1-In urban canyons, the satellites at across-street direction are likely to be blocked by buildings.

navigation and used to improve positioning performance in urban canyons. Satellite visibility can be predicted using $3 \mathrm{D}$ city models (3D geographic information systems or $3 \mathrm{D}$ maps) [3, 14-20]. Predicted satellite visibility by 3D city models can then be used for NLOS or multipath detection and elimination [13, 21-25]. The path delay of NLOS may also be modeled as a function of the user position, enabling NLOS signals to be used for position determination [23]. Furthermore, 3D city models are also applied to enhance map matching [24] and image matching in land vehicle navigation [26].

A new approach has recently be proposed to improve the cross-street accuracy using GNSS, assisted by knowledge derived from 3D building models close to the user of navigation devices $[2,27]$. In shadow matching, satellites visible from different locations are predicted and compared with the measured satellite visibility to determine position. A preliminary shadow-matching algorithm was developed that demonstrated the ability to distinguish pavement from vehicle lane, and identify the correct side of street using real-world GPS and GLONASS measurements [28, 29]. However, only direct line-of-sight (LOS) signals are predicted in the earlier algorithm, whereas the user equipment can also observe diffracted and reflected signals. This mismatch can degrade shadow-matching performance.

In this work, a number of improvements have been made. First, a new scoring scheme, a key element of the algorithm to weight candidate user locations, is proposed. The new scheme takes into account the effects of satellite signal diffraction and reflection by weighting the scores based on diffraction modeling and signal-to-noise ratio (SNR). Furthermore, an algorithm similar to k-nearest neighbors (k-NN) is developed to interpolate the position solution over an extensive grid. The process of generating this grid of building boundaries is also optimized. Finally, instead of just testing at two locations as in the earlier work, real-world GNSS data has been collected at 22 different locations in this work, providing a more comprehensive and statistical performance analysis of the new shadowmatching algorithm.

The improved shadow-matching algorithm is described in the second section, employing a set of new scoring schemes to acknowledge signal diffraction and reflection. The third section then describes the testing of the algorithm using realworld GPS and GLONASS measurements, and compares performance of the shadow-matching algorithm using different scoring schemes. Finally, conclusions are drawn and future work discussed.

\section{SHADOW MATCHING OPTIMIZATION}

This section describes the full implementation of the shadow-matching algorithm and discusses how it was optimized. We first explain the existing shadow-matching algorithm and then give a comprehensive implementation of the algorithm, which consists of two phases - the offline phase and online phase. Each step in the two phases is further introduced, with emphasis on optimization in grid generation of building boundaries and a set of proposed new scoring schemes.

\section{The Existing Shadow-Matching Algorithm}

The principle of shadow matching is simple [2]. Due to obstruction by buildings in urban canyons, signals from many GNSS satellites will be receivable in some parts of a street, but not others. Figure 2 


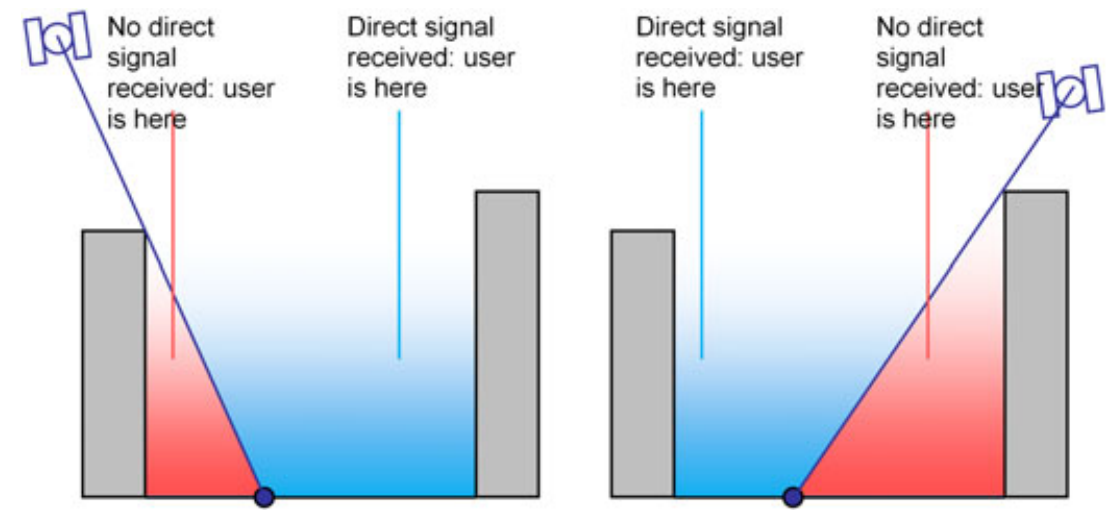

Fig. 2-The shadow-matching concept: using direct signal reception to localise position. [2]

illustrates this, noting that the boundary between the two regions is fuzzy due to diffraction effects at building edges [14]. Where each direct signal is receivable can be predicted using a $3 \mathrm{D}$ city model. Consequently, by determining whether a direct signal is being received from a given satellite, the user can localize their position to within one of two areas of the street. By considering other satellites, the position solution may be refined further. At each epoch, a set of candidate user positions is generated close to the user's low-accuracy conventional GNSS positioning solution. At each candidate user position, the predicted satellite visibility is matched with the real observations. The candidate position that has the best match between the prediction and the real observations is deemed the shadow matching positioning solution. This process can be conducted epoch by epoch, so the GNSS user can be either static or dynamic.

\section{The Improved Shadow-Matching Algorithm}

The new shadow-matching algorithm has two phases - the offline phase (the preparation step) and the online phase, consisting of five steps, both illustrated in Figure 3. An off-line phase is conducted to generate a grid of building boundaries. In the beginning of the online phase, the user position is first initialized, e.g., using standard point positioning (SPP) with GNSS pseudo-ranges. The second step defines the search area for the shadowmatching position solution. For the third step, the satellite visibility at each grid position is predicted using the building boundaries generated from the $3 \mathrm{D}$ city model. After that, the similarity of satellite visibility between prediction and observation is evaluated using a scoring scheme, providing a score for each grid point in the search area. Finally, the shadow-matching positioning solution is generated

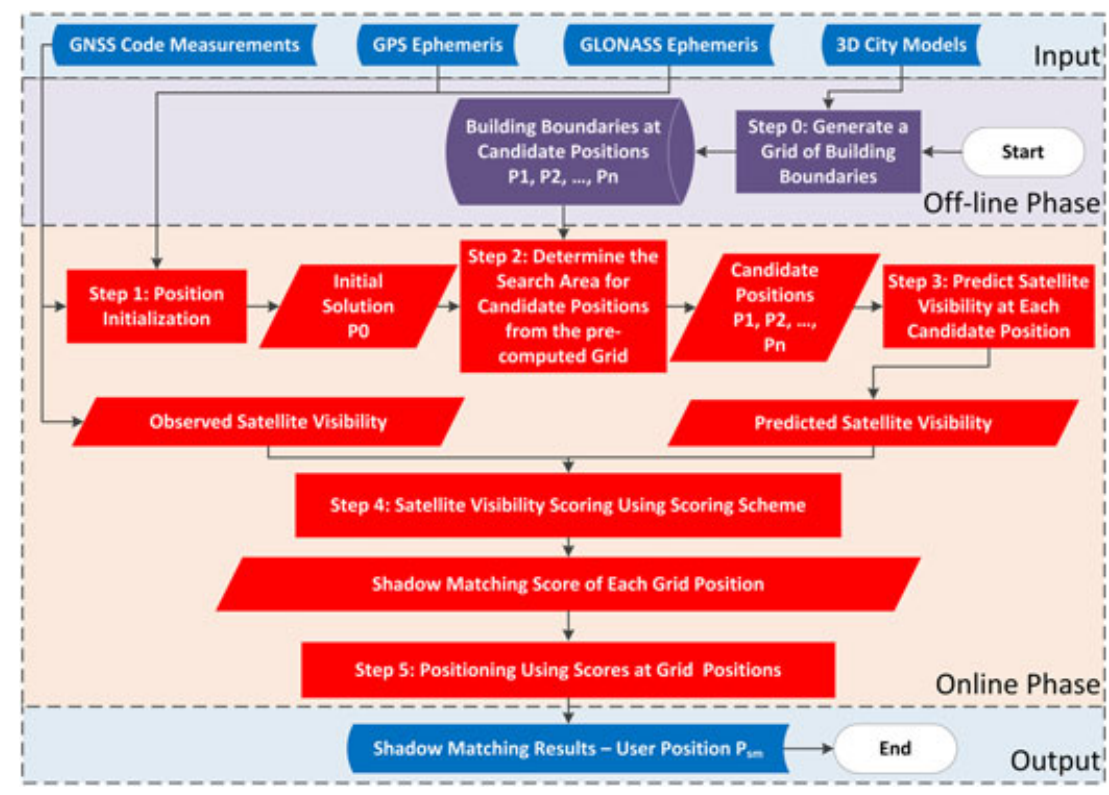

Fig. 3-A schematic diagram of shadow matching. 
by a modified k-nearest neighbors algorithm, which averages the grid points with the highest scores. Each of the steps is described in more detail below.

\section{- Step 0: Generate a Grid of Building Boundaries}

In the off-line phase, building boundaries at a grid of locations are generated. A building boundary means, from a GNSS user's perspective, that the building's edge is determined for each azimuth (from 0 to $360^{\circ}$ ) as a series of elevation angles. The results from this step show where the building edges are located within an azimuth-elevation sky plot. Once the building boundary has been computed, it may be stored and reused easily in the online phase to predict satellite visibility by simply comparing the elevation of a satellite with the elevation of the building boundary at the same azimuth.

From the perspective of mobile devices, limited computational power, memory, and battery life are of great importance. To overcome these limits, the proposed method is designed to move the most computationally intensive tasks from the mobile devices to the server. Thus, the designed algorithm exchanges real-time computational load for a one-off processing requirement at the server side. Specifically, this is achieved by representing the 3D model in a specially designed form - building boundaries at a grid of positions. The logic behind the strategy is that the vast amount of data in a 3D city model is not of direct interest to the shadowmatching algorithm, only the locations of the building edges matter from a user's perspective. Thus, utilizing this knowledge, only building boundaries at each candidate position are abstracted from the $3 \mathrm{D}$ model. This method saves computation load because individual mobile devices do not need to compute the building boundaries on the fly. Instead, they can simply request building boundaries at a certain range of locations, or cache a desired region.

Using stored building boundaries, fewer than fifty comparison and addition operations are required to calculate an overall shadow matching score for one candidate position with two GNSS constellations. Therefore, shadow matching may be performed in real time on a mobile device with several hundred candidate positions where necessary.

Without any data compression, about 300 bytes are required to store a building boundary with a $1^{\circ}$ resolution. If a $2 \times 2 \mathrm{~m}$ grid spacing is used for the candidate positions, a $1 \mathrm{~km}$ long $20 \mathrm{~m}$ wide street will contain 5000 grid points, requiring 1.5 MB of data storage. By exploiting the similarities both between neighboring azimuths in the same building boundary and between building boundaries at neighboring grid points, substantial data compression should be achievable; possibly up to a factor of ten.
Therefore, a standard 4 GB flash drive could store building boundary data for $2500-25000 \mathrm{~km}$ of road network. For comparison, the Greater London metropolitan area contains about $15000 \mathrm{~km}$ of road. However, as shadow matching is only useful in streets where conventional GNSS positioning is poor, the database need only contain building boundary data for these streets, maybe $10 \%$ of the total. Therefore, it should be practical to preload a mobile device with shadow-matching data for several cities, which could be kept up-to-date via the internet.

A software toolkit for generating the grid of building boundaries from a 3D city model was developed in $\mathrm{C}++$. Figure 4 shows the process.

The process can be broken into four steps. First, a one meter by one meter horizontal grid of points, covering the $3 \mathrm{D}$ city model area, is generated. The height is set to be $1.5 \mathrm{~m}$ above the terrain height measured in the 3D city model. Second, a preprocessing step is developed to eliminate indoor points from the generated grid in the first step, because the current shadow-matching algorithm is designed to work outdoors. Outdoor points are distinguished from indoor ones by testing whether the elevation angle of the sky at each azimuth is 90 degrees. Further details of the algorithms testing line-of-sight visibility can be found in a previous

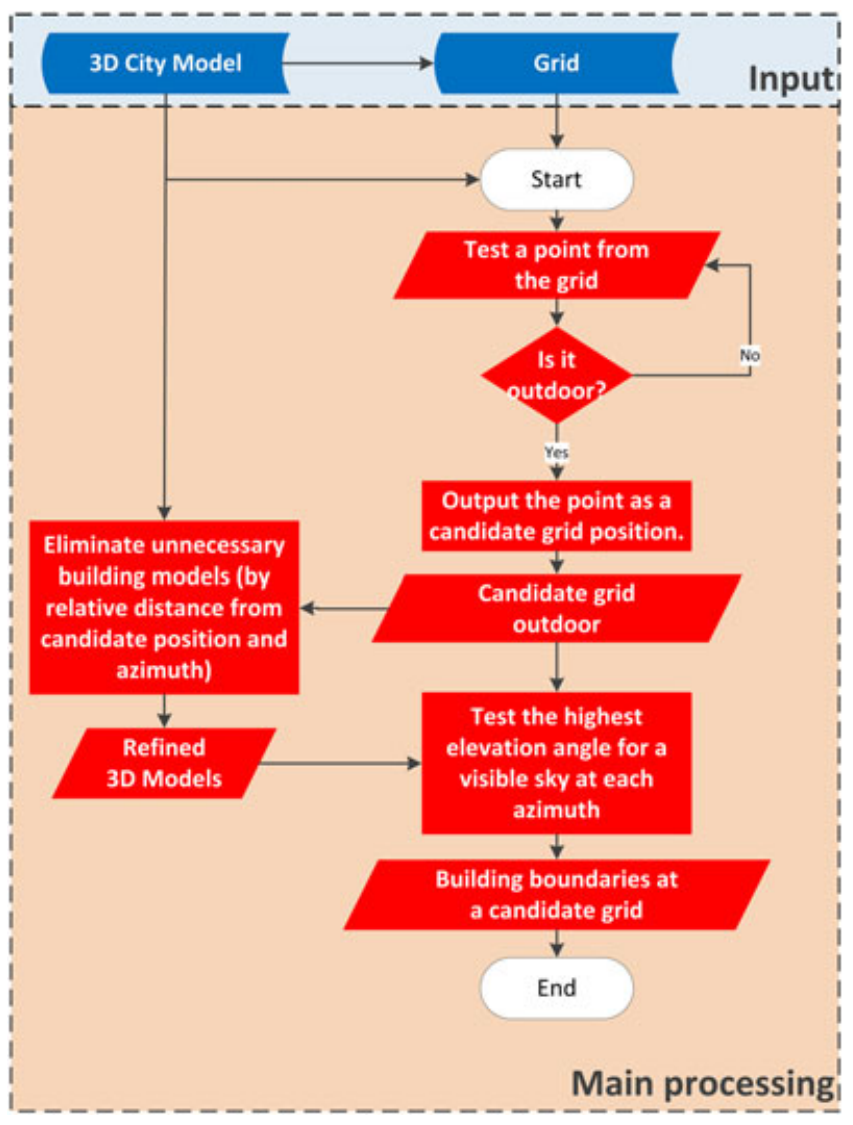

Fig. 4-The process generating the grid of building boundaries. 
paper [3]. Third, buildings that are unlikely to block satellite signals are eliminated from the search area, based on checks of their relative location from the candidate position of interest. Finally, the highest elevation angle for a visible sky at each azimuth is tested to determine the building boundary at each outdoor candidate position.

Figure 4 also illustrates the optimization of the process of building boundary generation. Without optimization, it takes an estimated 53 days to perform the process at a $1 \mathrm{~m}$ by $1 \mathrm{~m}$ grid of candidate positions across a $500 \mathrm{~m}$ by $500 \mathrm{~m}$ area, using a computer with a CPU speed of $2.67 \mathrm{GHz}$. In order to improve the efficiency, only buildings that are close to the candidate position and in the direction of interest are tested. Figure 5 illustrates this search area. It should be noted that the parameters used in this example are manually selected based on knowledge of the 3D city model used in this work. Appropriate changes should be made if using another type of city model. After optimization, the time required to generate building boundaries at the same grid of points was reduced to less than 4 days, a $92.5 \%$ reduction in time compared to the original algorithm.

\section{- Step 1: Position Initialization (Online Phase)}

In the first step of the shadow-matching algorithm, standard point positioning (SPP) using GNSS pseudo-ranges is conducted to acquire an initial user position. In an urban environment, the accuracy is often poor. Consistency checking may be used to identify non-line-of-sight signals and remove them from the position solution [1, 30]. Other available positioning methods (e.g., Wi-Fi or cell network solution) may be introduced into this step when the GNSS SPP is poor or unavailable.

- Step 2: Determine the Search Area for Candidate Positions from the Building Boundaries at a Grid

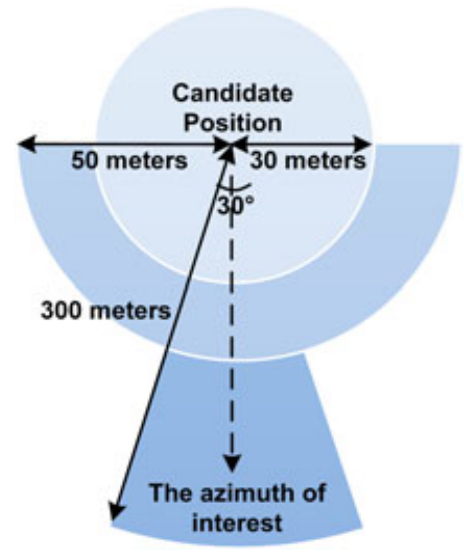

Fig. 5-The optimization used in building boundary generation by refining city models according to location of candidate user position and azimuth of interest. (Aerial perspective, the figure is not drawn to scale).
The second step defines the search area in which candidate positions are located for the shadowmatching position solution. A search area is defined based on an initial position generated in the first step. In the current implementation, the search area comprises the area within a fixed-radius circle centered at the initialized position. Indoor locations are excluded from the search area where the building boundaries grid is generated in the offline phase. For the version of shadow matching presented here, the circle radius was determined empirically. However, more advanced algorithms can be developed to use the knowledge from the initialization process to optimize the search area.

For instance, if the initial position is generated using a conventional GNSS solution, the signal geometry, and hence the positioning accuracy, will be much better along the direction of the street than across the street. This is because an urban canyon affects the geometry of the available GNSS signals. Signals with lines of sight going across the street are much more likely to be blocked by buildings than signals with lines of sight running along the street. Therefore, the conventional GNSS solution has lower accuracy across-street and higher accuracy along-street, which is complementary to the shadow-matching algorithm.

Thus, the along-street component of the SPP solution can be used as a reference to define the search area and thus generate candidate user positions that vary more in the across-street direction. This is illustrated in Figure 1, with the green area representing the search area centered at the initial position and the grid representing candidate positions. A more advanced shadowmatching algorithm would vary the size of its search area based on an assessment of the quality of the initial position.

- Step 3: Predict Satellite Visibility at Each Candidate Position

In the third step, performed at each candidate position, each satellite's elevation is compared with the building boundary elevation at the same azimuth. When a satellite elevation is below the building boundary, the buildings block any satellite signals, assuming there are no holes in them allowing signals to travel through. Thus, the satellite is predicted to be visible if the satellite is above the building boundary; otherwise, the satellite is predicted to be invisible. This is illustrated in (1) and Figure 6.

$$
V_{s, p}= \begin{cases}1, & \theta_{n u}^{a s} \geq \theta_{n u}^{p b}\left(\varphi_{n u}^{a s}\right) \\ 0, & \theta_{n u}^{a s}<\theta_{n u}^{p b}\left(\varphi_{n u}^{a s}\right)\end{cases}
$$

where $\boldsymbol{V}_{\boldsymbol{s}, \boldsymbol{p}}$ denotes the predicted visibility of the satellite $\boldsymbol{s}$ at the candidate position $\boldsymbol{p}, \boldsymbol{\theta}_{n u}^{a s}$ denotes 


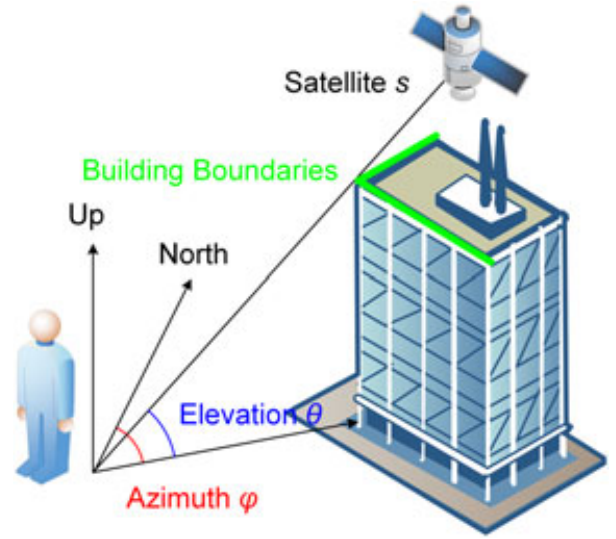

Fig. 6-Illustration of building boundaries, azimuth elevation angle.

the elevation of satellite $\mathbf{s}$, $\boldsymbol{a}$ denotes user antenna, $\mathbf{n}$ denotes the local navigation frame, $\mathbf{u}$ denotes line-of-sight unit vector, and $\boldsymbol{\theta}_{n u}^{p b}\left(\varphi_{n u}^{a s}\right)$ denotes the elevation of building boundary at azimuth $\varphi$, from the perspective of the user position [12].

The diffraction effect is also modeled in this work. A three-degree diffraction zone is modeled for building boundaries both horizontally and vertically [3]. Thus, in this model, from the perspective of a GNSS receiver, buildings are three degrees lower and narrower than their actual height and width. If the line-of-sight (LOS) falls within the diffraction region, the signal is predicted to be diffracted. Otherwise, it is predicted to be invisible. This is illustrated in Figure 7, with the blue lines representing the building boundaries and the red lines representing the diffraction region.

- Step 4: Satellite Visibility Scoring Using Scoring Scheme

For the fourth step, the similarity of the satellite visibility between predictions and observations is evaluated. The candidate positions with the better matches will then be weighted higher in the shadow matching positioning solution. There are two stages

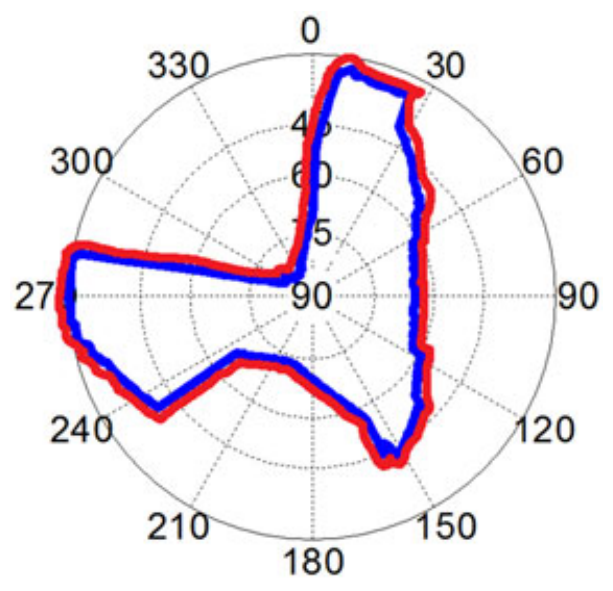

Fig. 7-An example of diffraction modelling. for calculating a score for a candidate position. First, each satellite above the elevation mask angle is given a score, calculated based on the predicted and observed visibility, using a scoring scheme. Second, the position scoring function, evaluates the overall degree of match between predicted and observed satellite visibility for each possible user position. This is illustrated in (2).

$$
f_{p o s}(p)=\sum_{i=1}^{m} f_{\text {sat }}(s, p, S S)
$$

where $f_{\text {pos }}(p)$ is the position score for grid point $p, f_{\text {sat }}$ $(s, p)$ is the score of satellite $s$ at grid point $p, m$ is the number of satellites above the mask elevation angle, and $S S$ is the scoring scheme which defines a score based on predicted and observed satellite visibility.

By the end of this step, each candidate position should have a score to represent the degree to which it matches the observed satellite visibility, and thus how likely it is that each candidate position is close to the true location.

The existing scoring scheme $S S_{22}$ is shown in Figure 8a. Only direct line-of-sight (LOS) signals are considered using this scoring scheme, whereas the user equipment can also observe diffracted and reflected signals. This mismatch can degrade shadow-matching performance.

Thus, the scoring scheme has been improved to acknowledge diffraction effects via diffraction modeling. Diffraction occurs at the edge of a building (or other obstacle) when the incoming signal is partially blocked, noting that the path taken by a GNSS signal is several decimeters wide. There are two approaches to predicting the effect of diffraction on satellite visibility using a $3 \mathrm{D}$ city model. The first one would be to numerically determine the diffraction field based on every physical factor, including the surface of the building, the angle of incidence of the signal, and the properties of the GNSS user equipment. This method is impractical because the necessary information about the building materials and antenna characteristics is difficult to obtain and the computational complexity is high. The second, much simpler, approach has been adopted here. This simply extends the building boundary used for satellite visibility determination by adding a diffraction region to model the diffraction effect around the building edge. Thus, wherever the LOS intersects the diffraction region, the signal is classified as potentially diffracted instead of blocked [3, 14, 31]. Both horizontal and vertical edges are considered for diffraction modeling. Here, a $3^{\circ}$-wide diffraction region was modeled. The improved scoring scheme $S S_{23}$ is shown in Figure 8b.

As diffractions and reflections both normally result in weaker signal reception, the signal strength is also built into the new scoring scheme $-S S_{32}$, as shown in 

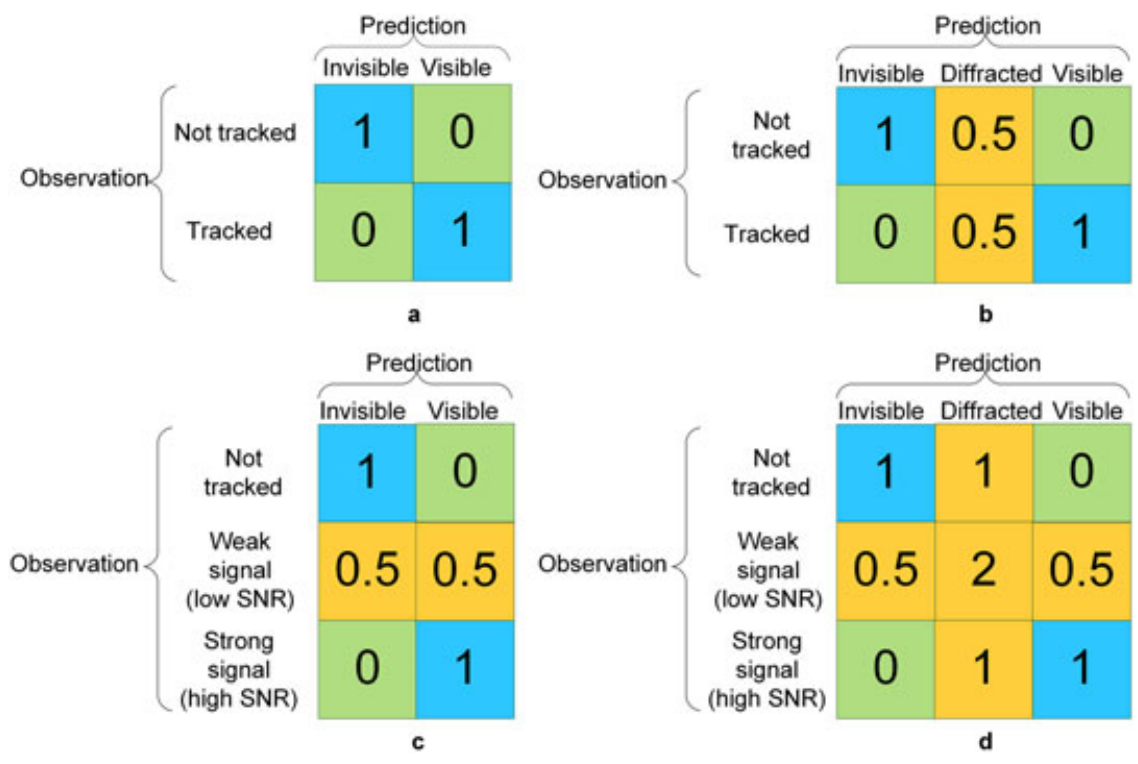

Fig. 8-Scoring matrix giving the score for each satellite in shadow matching.

Figure 8c. In this scheme, a weak signal is deemed likely to be reflected or diffracted, thus it is given a lower weight compared to a strong signal. The boundary to distinguish weak signal from strong signal should be based on the signal to noise ratio (SNR).

Finally, by joining both diffraction modeling and signal strength based scoring, a new $S S_{33}$ scoring scheme is introduced, as shown in Figure 8d. It should be noted that the scores in these scoring schemes are based on both theory and experimental data. We believe that a high weight for weak signals predicted to be diffracted gives good results because diffracted signals are typically weaker than direct line of sight. Weak signals that are not predicted to be diffracted are given a low weighting because they are likely to be NLOS signals that cannot currently be predicted. Changes to the scores may be needed when using GNSS receivers of other types.

In the third section, a comprehensive comparison will be conducted to evaluate the influence of different scoring schemes on the performance of shadow matching.

- Step 5: Positioning Using Scores at Candidate Positions

The last step of the shadow-matching algorithm is to generate a positioning solution using scores from each candidate position. Shadow matching uses the pattern-matching positioning method [12]. As the process of Wi-Fi fingerprinting is similar to the process in shadow matching, the algorithms used in Wi-Fi fingerprinting may be investigated for their potential implementation in shadow matching. Potential algorithms include, but are not limited to, k-weighted nearest neighbors, the Bayesian inference received signal strength (RSS) location method, and the particle filter.
In this work, a method similar to k-nearest neighbors is used to estimate the location, averaging the grid positions of highest scores. With the current scoring system, scores take integer or half-integer values. Therefore, several grid points typically share the highest score. The points in the grid with highest scores are thus equivalent to the nearest neighbors. For $l$ highest-scoring points, the location estimate is determined using (3) and (4) for the northing and easting projected coordinate components, $N_{a}$ and $E_{a}$, respectively:

$$
\begin{aligned}
N_{a} & =\frac{1}{l} \cdot \sum_{i=1}^{l} N_{i} \\
E_{a} & =\frac{1}{l} \cdot \sum_{i=1}^{l} E_{i}
\end{aligned}
$$

where $N_{i}$ and $E_{i}$ are, respectively, the northing and easting coordinates of the $i^{t h}$ high-scoring candidate positions. Note that $l$ varies from epoch to epoch depending on how many candidate positions share the highest score.

\section{COMPARISON OF VISIBILITY PREDICTION SCORING USING EXPERIMENTAL DATA}

The different scoring schemes were tuned and compared using experimental data to improve the accuracy and reliability of shadow matching. The next subsection introduces the 3D city model of the Aldgate area of central London, used in the shadow matching experiments. Real-world data sets are collected at sites within the city model area, scattered on major roads and minor roads, at and between junctions. Subsequent text describes the methods and logics behind implementations of each step of shadow matching with details of 
selected experimental sites coming next. The experimental results are compared and analyzed in the final subsections.

\section{City Models}

A real 3D city model of the Aldgate area of central London, supplied by ZMapping, Ltd has been used. The model has a high level of detail and decimeterlevel accuracy. Figure 9 shows an aerial view of the city model used in this work.

The software toolkit developed for this study stores and processes 3D city model data using Virtual Reality Modeling Language (VRML), an international standard format. Model data in other formats can be transformed to VRML. Buildings in VRML format are represented by structures, which in turn comprise polygons (normally triangle meshes).

\section{Shadow Matching Implementation}

In the offline phase, a $1 \mathrm{~m}$ by $1 \mathrm{~m}$ grid has been generated, and the building boundaries determined at each grid point as defined earlier in the paper. They are stored in a specially defined format in a database.

In the online phase, position initialization is not conducted because this study focuses on comparing the different scoring schemes. Different methods used in positioning initialization can result in very different initial positions, so in order to prevent initialization errors from contaminating the following scoring step, the search area for each site is centered at the true position. The search area for each site is defined as everything within a radius of $20 \mathrm{~m}$, except for the indoor points. Four scoring schemes are deployed at every site in the satellite visibility scoring step. The modified k-nearest neighbors algorithm is used to determine the positioning solution of the shadow-matching algorithm using (3) and (4).

\section{Experimental Site Selection}

To compare the performance of shadow matching using different scoring schemes, experiments were conducted at 11 pairs of sites, resulting in GNSS data at 22 locations in central London on 23/07/ 2012. In each pair, two survey-grade GNSS receivers (Leica Viva) were set up on opposite sides of each street (Leadenhall Street, Billiter Street, and Fenchurch Street), standing on a footpath close to the traffic lane. GPS and GLONASS observation data were recorded at a $1 \mathrm{~Hz}$ rate simultaneously for $10 \mathrm{~min}$ at each pair of locations. For the purpose of increasing the reliability of the experiments, each site was visited twice at an interval of approximately $4 \mathrm{hr}$, allowing the satellite geometry to change completely. The first round is denoted $\mathrm{r} 1$, the second round is denoted $\mathrm{r} 2$. Thus, in total, $7 \mathrm{hr}$ and $20 \mathrm{~min}$ of GNSS data was recorded in 44 observation periods at 22 different locations. A summary of the experimental sites is shown in Table 1; their locations are presented in Figure 10.

\section{Signal to Noise Ratio (SNR) Empirical Value}

The signal to noise ratio (SNR) is introduced as an indicator of satellite signal quality in the shadow-matching

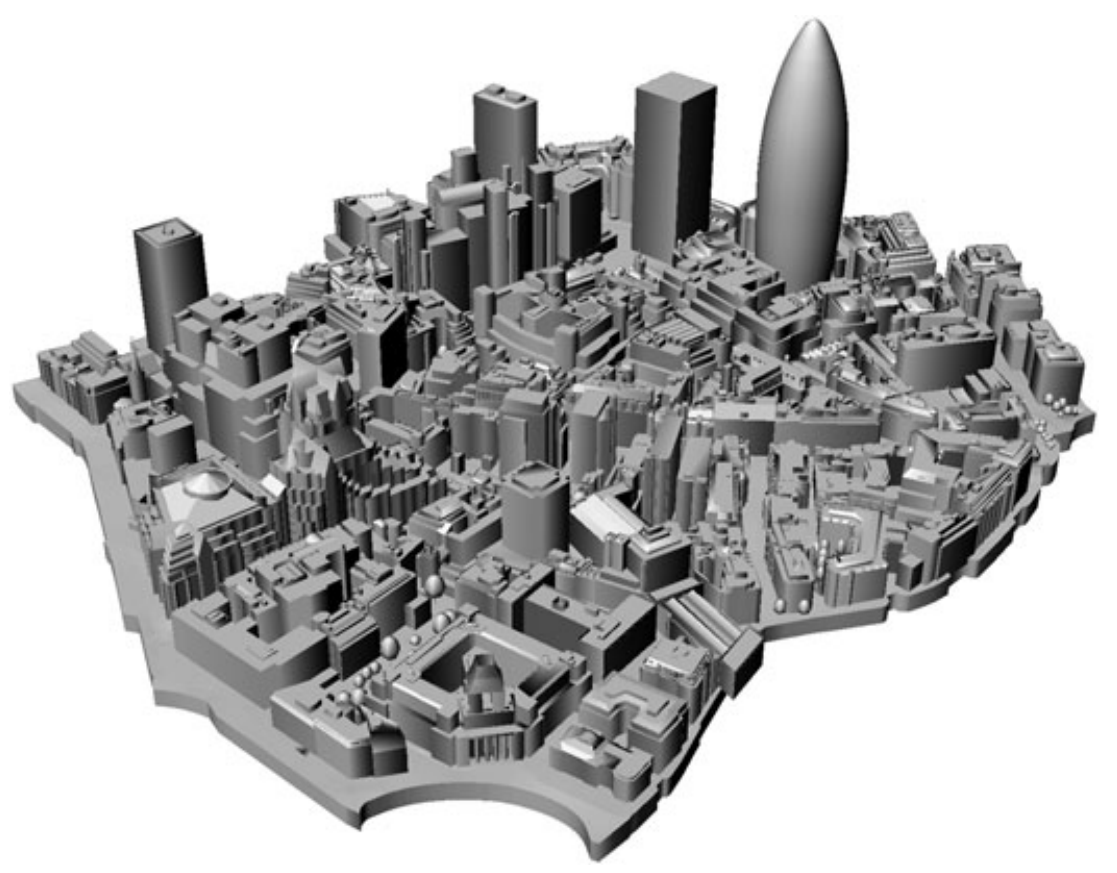

Fig. 9-Part of the 3D model of London used in the experiments. 
Table 1-A summary of experimental sites

\begin{tabular}{lcc}
\hline Site Name & 1st Round & 2nd Round \\
\hline G001, R001 & $09: 05-09: 15$ & $13: 07-13: 17$ \\
G002, R002 & $09: 35-09: 45$ & $13: 19-13: 29$ \\
G003, R003 & $09: 10-10: 00$ & $13: 31-13: 41$ \\
G004, R004 & $10: 05-10: 15$ & $13: 44-13: 54$ \\
G005, R005 & $10: 18-10: 28$ & $13: 58-14: 08$ \\
G006, R006 & $10: 33-10: 43$ & $14: 11-14: 21$ \\
G007, R007 & $10: 45-10: 55$ & $14: 23-14: 33$ \\
G008, R008 & $10: 59-11: 09$ & $14: 36-14: 46$ \\
G009, R009 & $11: 14-11: 24$ & $14: 49-14: 59$ \\
G010, R010 & $11: 31-11: 41$ & $15: 03-15: 13$ \\
G011, R011 & $11: 47-11: 57$ & \\
\hline
\end{tabular}

system. An empirical analysis was first conducted to observe the level of SNR in the experimental data. This is because SNR can vary significantly between different types of GNSS receiver. The SNR of the L1 C/A code signal recorded by the Leica Viva GNSS receiver is shown in Figure 11. Figure 11a shows a period of observation with typical 'strong' SNR values; Figure $11 \mathrm{~b}$ shows the same period of observation, but with typical 'weak' SNR values. The figure also shows that when the signal is strong, the SNR value typically remains stable (normally around $50 \mathrm{~dB}-\mathrm{Hz}$ ), whereas when the signal is weak, it changes dramatically and the value tends to be lower (normally below $40 \mathrm{~dB}-\mathrm{Hz}$ ).

SNR values of all satellites recorded by two identical Leica Viva receivers in the experimental period show that the SNR mainly ranges between $25 \mathrm{~dB}-\mathrm{Hz}$ and $55 \mathrm{~dB}-\mathrm{Hz}$ with an average of $40 \mathrm{~dB}-\mathrm{Hz}$. Thus, in those scoring schemes that account for the

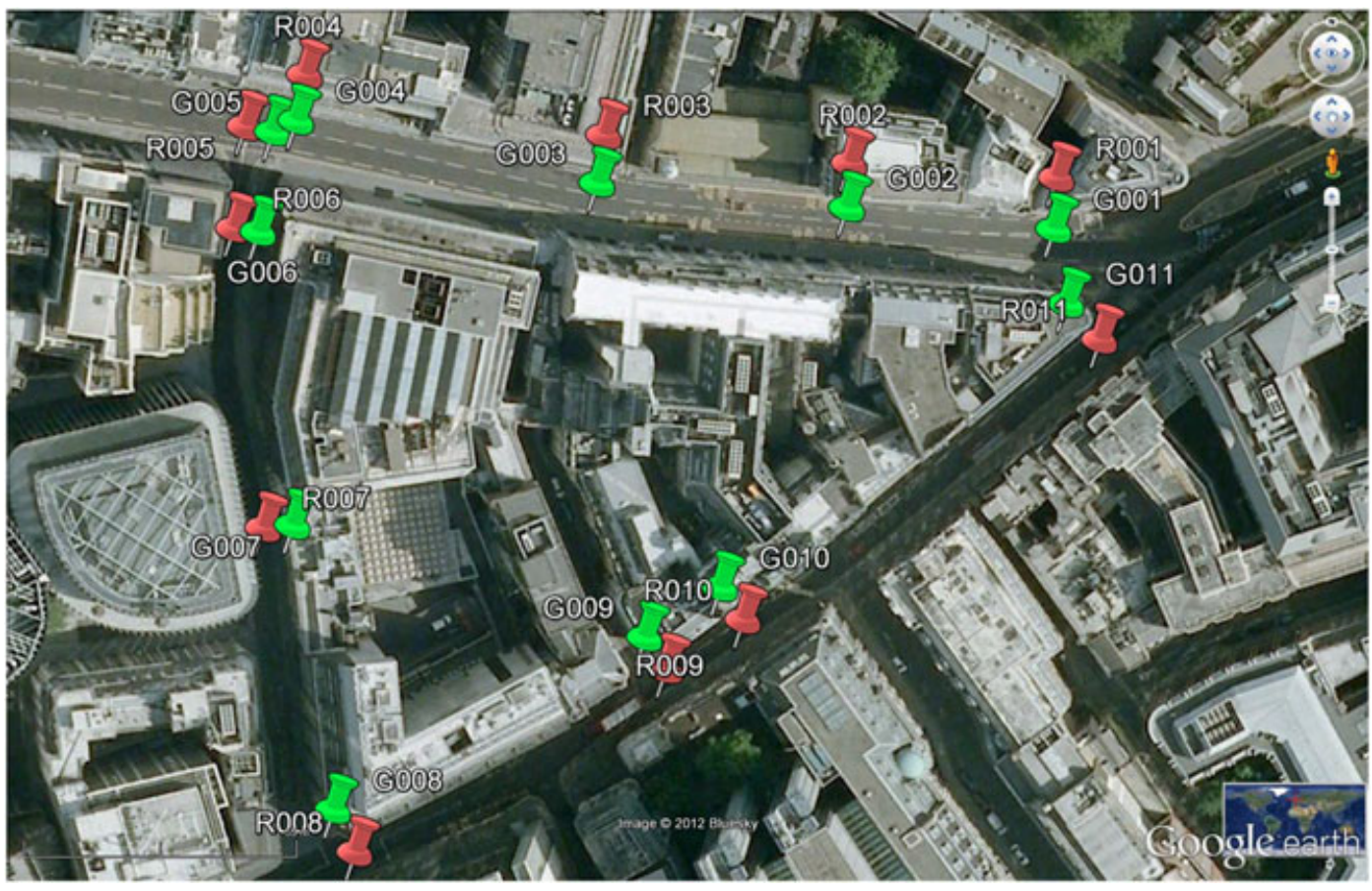

Fig. 10-The experimental sites in urban canyons. It shows the experimental sites location in the satellite image in real world.

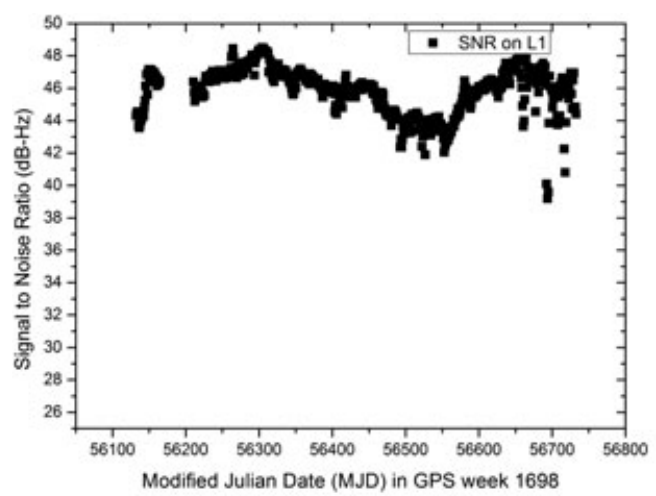

a

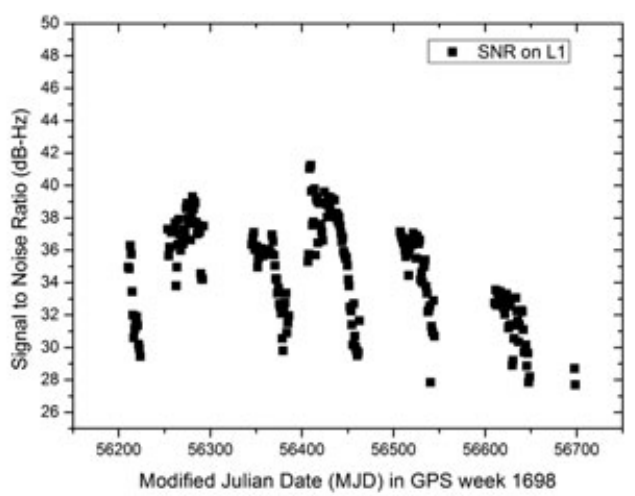

b

Fig. 11-a: A period observation of typical strong signal (SNR on L1 of GPS PRN 2, on experimental site ID G001_r1); b: A period observation of typical weak signal (SNR on L1 of GLONASS 18, on experimental site ID G001_r1). 
observed signal quality, signals with $\mathrm{SNR}>40 \mathrm{~dB}-\mathrm{Hz}$ are regarded as strong and signals with $\mathrm{SNR} \leq 40$ $\mathrm{dB}-\mathrm{Hz}$ are regarded as weak.

\section{Score Map of Candidate Positions}

At the true position of each experimental site, a $20 \mathrm{~m}$ radius circle is used to generate candidate positions. The pre-calculated candidate grid of building boundaries is loaded in the on-line phase of shadow matching. At each observation epoch, comparison is made between the predicted and observed satellite visibility. Each of the four score schemes is applied to the results for comparison. To illustrate the distribution of scores at the grid points, Figure 12 shows an example of score map for experimental sites G011 (left) and R011 (right).

In Figure 12, the score of candidate positions ranges mainly in the across-street direction. As G011 and R011 are located at different sides of a street, it is clearly demonstrated that the shadow matching algorithm is sensitive to changes in the across-street direction, but less sensitive in the along-street direction. This is in line with expectations and complements conventional GNSS positioning, which is generally more precise in the along-street direction. There are some locations where the space between buildings falls within the search area, but the highest scoring points are mostly on the correct street. In order to evaluate the performance across all of the experimental data, statistical analysis was conducted.

\section{Statistical Analysis}

Mean absolute deviation is used for each experimental site to evaluate the performance of shadow matching. This is transformed from local coordinates (Northing and Easting) to the along street and across street directions. In order to compare shadow matching using the different scoring schemes, the mean absolute deviation at each site is shown for each scoring scheme in Figure 13. It should be noted that the statistics cover a 10 min observation period, during which the constellation geometry changes slowly, so the results are highly correlated over time. When separate statistics are calculated for the two different observation periods at the same site, results may be considered independent. Therefore, these are shown separately in Figure 13. A few sites are missing from the results because fewer than four satellites were observed, meaning that an SPP solution could not be computed and the GNSS receivers used for this experiment would not record the measurements due to the design of their software.

It is shown in Figure 13 that the along street absolute mean deviation is typically higher than the across street one. As shadow matching was designed to improve the across-street positioning, and may be combined with conventional GNSS and other possible techniques [32], this is not considered to be a problem.

Further statistics have been computed to average the mean absolute deviation using each scoring scheme. The results are shown in Figure 14. Although the along-street performance in Figure 14 using a $3 \times 2$ scoring scheme is better than other scoring schemes, the across-street accuracy is more important because that is where conventional GNSS performance is poor.

Similarly, Figure 15 also compares different scoring schemes for their effects on shadow matching performance in terms of success rate for achieving across-street positioning error within a certain number of meters. It can be seen from both graphs that the different scoring schemes have a relatively small influence on the performance of shadow matching, which means that shadow matching

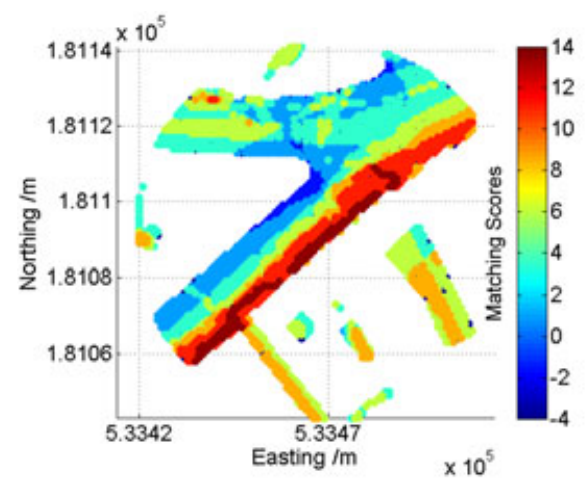

a

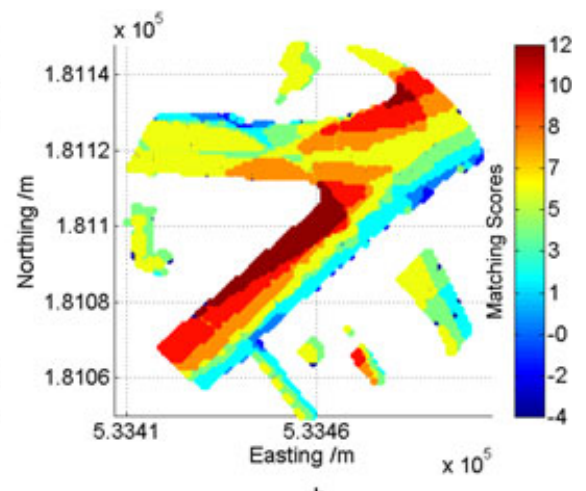

b

Fig. 12-Shadow-matching score map of experimental sites G011 (a) and R011 (b) using $3 \times 3$ scoring scheme SS33 (at epoch 11:55:40 23 July 2012). The circles represent the candidate positions. The red bar is where the shadow-match positioning solution is. Refer to Fig. 10 for the true location of each site. For illustration purposes, a 50 meter-radius circular search area centered at each truth position is used. 


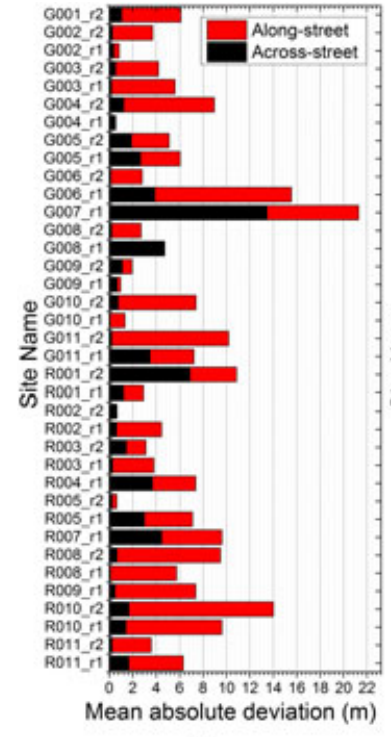

a

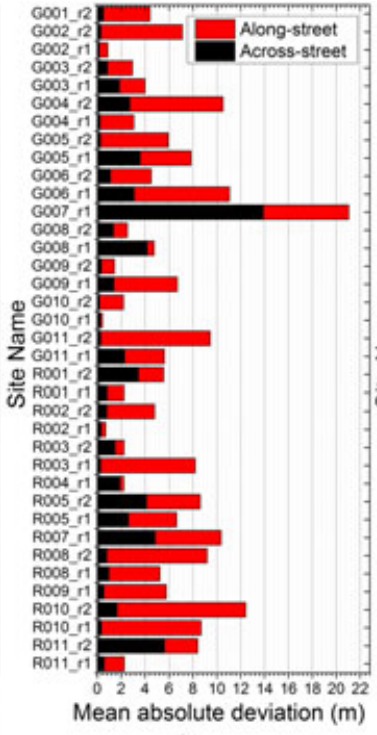

b

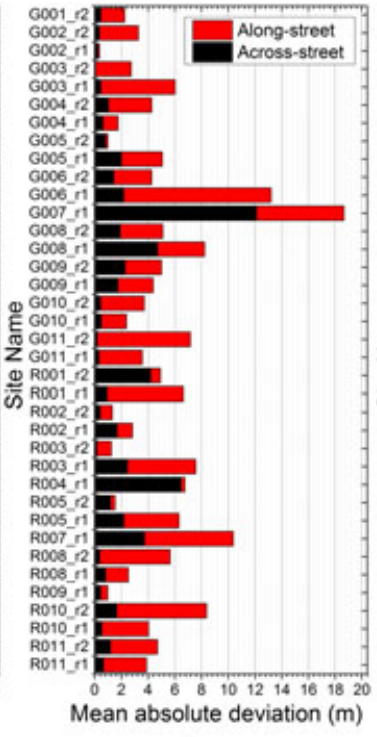

C

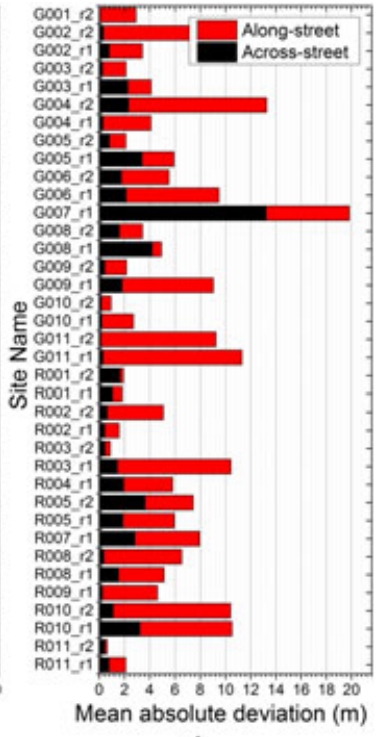

d

Fig. 13-The mean absolute deviation of shadow matching positioning solution for each experimental sites using the $2 x$ 2 (a), $2 \times 3$ (b), $3 \times 2$ (c), $3 \times 3$ (d) scoring scheme.

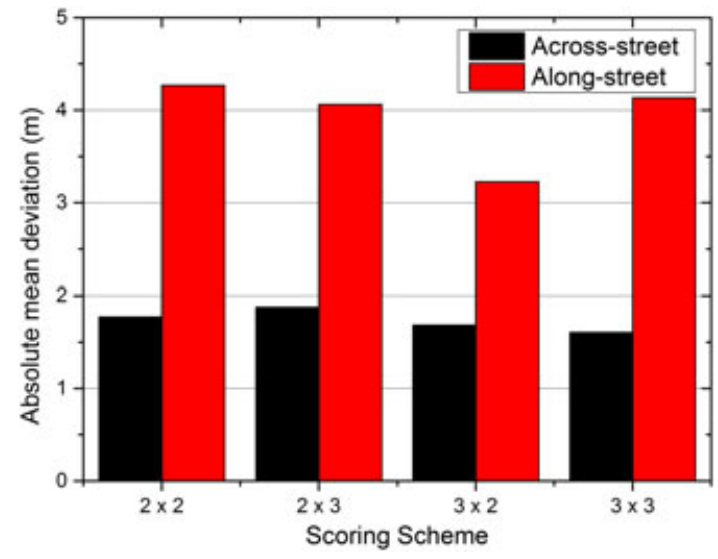

Fig. 14-The mean absolute deviation that is averaged between all experimental sites, using different scoring scheme in shadow matching algorithm.

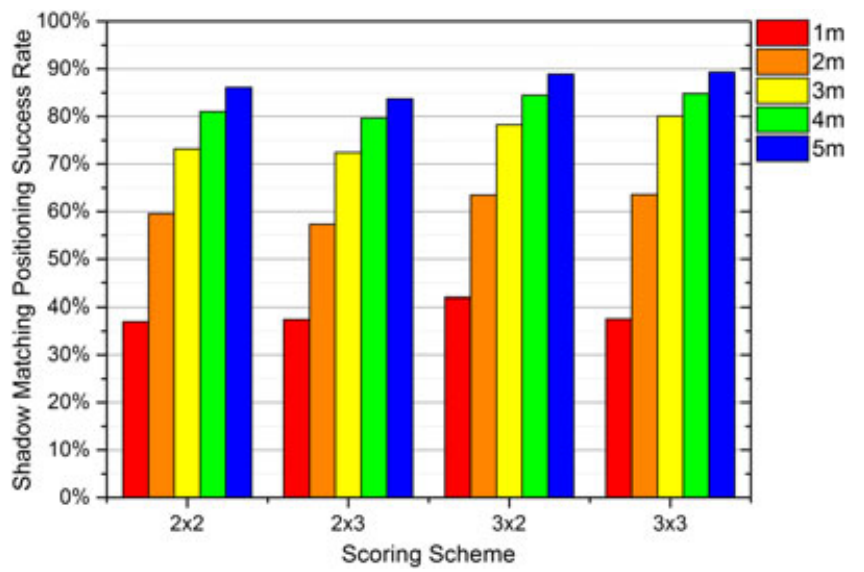

Fig. 15-The success rate of positioning error using shadow matching in cross-street direction. performance is not very sensitive to the scoring schemes. However, there is a small improvement using the new $S S_{33}$ scoring scheme. For example, in Figure 14, the new scoring scheme improves the across street accuracy with an average mean absolute deviation of $1.61 \mathrm{~m}$, with a $9.4 \%$ reduction compared to the original $S S_{22}$ scoring scheme.

As the street is around $10 \mathrm{~m}$ wide, a positioning accuracy better than $5 \mathrm{~m}$ is considered good enough to determine the correct side of the street, while a positioning accuracy better than $2 \mathrm{~m}$ is considered good enough to distinguish the footpath from a traffic lane. Figure 15 shows success rate in terms of achieving across-street error within 1, 2, 3, 4, and $5 \mathrm{~m}$. It shows that the success rate for determining the correct side of a street is $89.3 \%$ with the $S S_{33}$ scoring scheme being $3.6 \%$ better than using the previous $S_{2}$ scheme; the success rate of distinguishing the footpath from a traffic lane is $63.6 \%$ of the time with $S S_{33}$ being $6.8 \%$ better than using $S_{22}$.

\section{CONCLUSIONS AND FUTURE WORK}

This paper presents the first shadow-matching results with an extensive search grid. An algorithm similar to k-nearest neighbors (k-NN) has been developed to interpolate the position solution over the grid. A new scoring scheme, a key element of the algorithm to weight candidate user locations, has been proposed. The new scheme takes into account the effects of satellite signal diffraction and reflection by weighting the scores based on diffraction modeling and signal-to-noise ratio (SNR). The process of generating this grid of building boundaries has also 


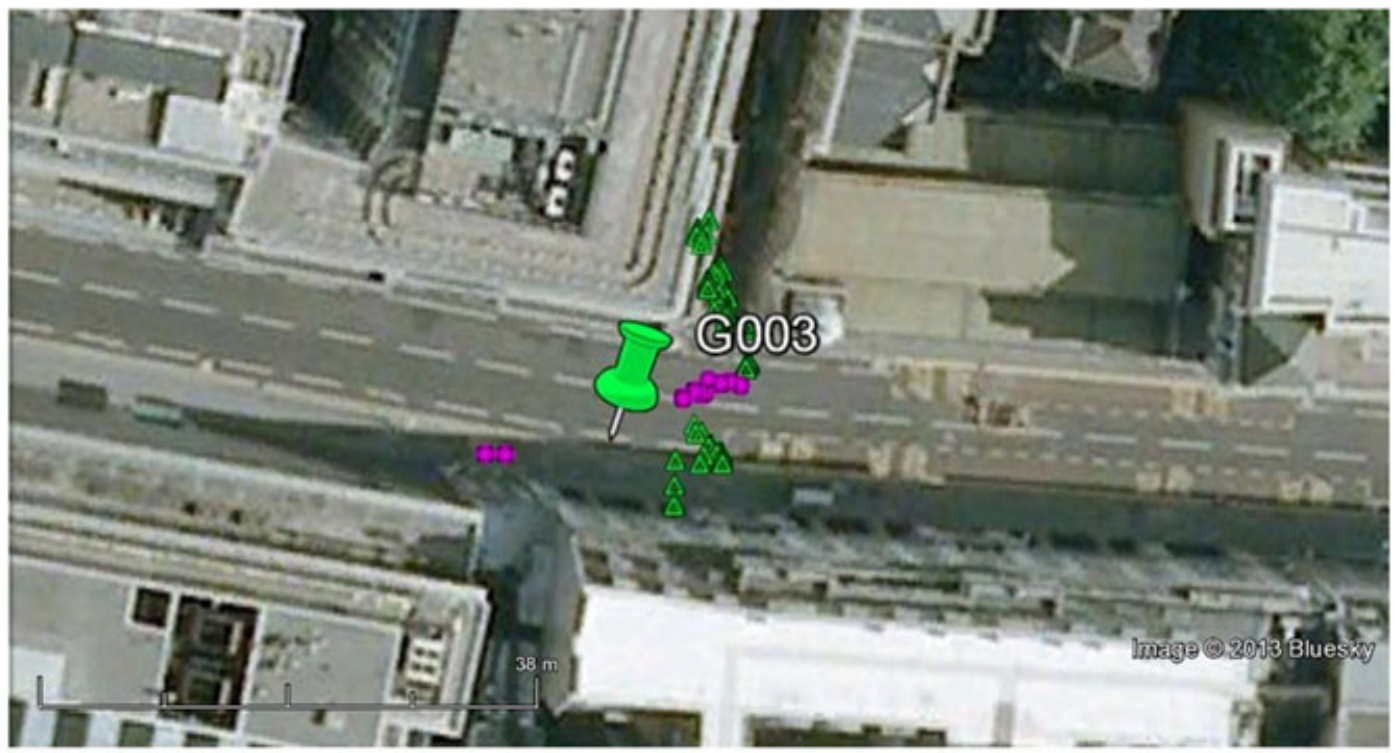

Fig. 16-Conventional GNSS positioning solution using weighted least square (WLS) at site G003 (green triangles denote the WLS solutions; purple circles denote the shadow matching solutions).

been optimized to reduce the computational load. Real-world GNSS data has been collected at 22 different locations in this work, providing a comprehensive and statistical performance analysis of the new shadow-matching algorithm.

In the experimental verification, the new scoring scheme achieves an average cross street accuracy of $1.61 \mathrm{~m}$, a $9.4 \%$ improvement over the previous scheme. The success rate for determining the correct side of a street is $89.3 \%$, a $3.6 \%$ improvement, while the success rate for distinguishing the footpath from a traffic lane is $63.6 \%$, a $6.8 \%$ improvement.

Conventional GNSS positioning performs relatively poorly in the across street direction, and better along the street. Figure 16 compares the conventional GNSS positioning solution using weighted least square (WLS) with shadow matching solutions at point G003_r1. It shows that the across-street positioning error of the conventional GNSS solution (WLS) can reach $20 \mathrm{~m}$, whereas the across-street positioning error of shadow matching solutions is less than $5 \mathrm{~m}$. As shadow matching has across-street accuracy of only a few meters, it is highly complementary to conventional GNSS positioning methods.

In future work, shadow matching using GPS and GLONASS data from a smartphone will be tested. Four-constellation shadow-matching performance will also be predicted by combining GPS and GLONASS data from two different epochs, separated in time. The Bayesian inference received signal strength (RSS) location method and the particle filter may be investigated for the shadow matching positioning algorithm. Further investigations will be conducted to improve the shadow-matching algorithm.

To obtain an accurate and reliable position solution in challenging urban environments, shadow matching must be combined with conventional GNSS positioning, NLOS signal detection, and other techniques that exploit the 3D mapping, such as height aiding. This concept is known as intelligent urban positioning (IUP) and is introduced in [32]. IUP may also be extended to incorporate other techniques, such as Wi-Fi, Bluetooth Low Energy, and MEMS inertial sensors.

\section{ACKNOWLEDGEMENTS}

The authors gratefully acknowledge Dr. Ziyi Jiang for his support with the experiments. This work has been jointly funded by the University College London Engineering Faculty Scholarship Scheme and the Chinese Scholarship Council. A version of this paper was originally presented at ION GNSS 2012, Nashville, Tennessee, September 17-21, 2012.

\section{REFERENCES}

1. Jiang, Z., Groves, P. D., Ochieng, W. Y., Feng, S., Milner, C. D., and Mattos, P. G., "Multi-Constellation GNSS Multipath Mitigation Using Consistency Checking," Proceedings of the $24^{\text {th }}$ International Technical Meeting of the Satellite Division of The Institute of Navigation (ION GNSS 2011), Portland, OR, September 2011, pp. 3889-3902.

2. Groves, P. D., "Shadow Matching: A New GNSS Positioning Technique for Urban Canyons," The Journal of Navigation, Vol. 64, 2011, pp. 417-430.

3. Wang, L., Groves P. D., and Ziebart, M. K., "MultiConstellation GNSS Performance Evaluation for Urban Canyons Using Large Virtual Reality City Models" The Journal of Navigation, Vol. 65, No. 3, 2012, pp. 459-476. 
4. Bruner, J., Greening the Traffic Lights, 2008 [cited February 23, 2013]; Available from: http://www. forbes.com/forbes/2008/0505/064.html.

5. Mountain, D., and Raper, J., "Positioning Techniques for Location-Based Services (LBS): Characteristics and Limitations of Proposed Solutions," Aslib Proceedings, 2001.

6. McCall, J. C., and Trivedi, M. M., "Video-Based Lane Estimation and Tracking for Driver Assistance: Survey, System, and Evaluation," IEEE Transactions on Intelligent Transportation Systems, Vol. 7, No. 1, 2006, pp. 20-37.

7. Dhar, S., and Varshney, U., "Challenges and Business Models for Mobile Location-Based Services and Advertising," Communications of the ACM, Vol. 54, No. 5, 2011,pp. 121-128.

8. Guy, R. and Truong, K., "CrossingGuard: Exploring Information Content in Navigation Aids for Visually Impaired Pedestrians," Proceedings of the SIGCHI Conference on Human Factors in Computing Systems, 2012 ACM, Austin, Texas, 2012, pp. 405-414.

9. Ercek, R., DeDoncker, P., and Grenez, F., "Statistical Determination of the PR Error Due to NLOS-Multipath in Urban Canyons," Proceedings of the 19th International Technical Meeting of the Satellite Division of The Institute of Navigation (ION GNSS 2006), Fort Worth, TX, September 2006, pp. 1771-1777.

10. Viandier, N., et al., "GNSS Performance Enhancement in Urban Environment Based on Pseudo-Range Error Model," Proceedings of IEEE/ION PLANS 2008, Monterey, CA, May 2008, pp. 377-382.

11. Farrell, J. A., Aided Navigation: GPS with High Rate Sensors, New York: McGraw-Hill Professional, 2008.

12. Groves, P., Principles of GNSS, Inertial, and Multisensor Integrated Navigation Systems (2nd edition), Boston, London: Artech House, 2013

13. Bradbury, J., et al., "Code Multipath Modelling in the Urban Environment Using Large Virtual Reality City Models: Determining the Local Environment," The Journal of Navigation, Vol. 60, No. 1, 2007, pp. 95-105.

14. Bradbury, J., "Prediction of Urban GNSS Availability and Signal Degradation Using Virtual Reality City Models," Proceedings of the 20th International Technical Meeting of the Satellite Division of The Institute of Navigation (ION GNSS 2007), Fort Worth, TX, 2007, pp. 2696-2706.

15. Suh, Y., and Shibasaki, R., "Evaluation of SatelliteBased Navigation Services in Complex Urban Environments Using a Three-Dimensional GIS," IEICE Transactions on Communications, Vol. E90-B, No. 7, 2007, pp. 1816-1825.

16. Kim, H. I., Park, K. D., and Lee, H. S., "Development and Validation of an Integrated GNSS Simulator Using 3D Spatial Information," Journal of the Korean Society of Surveying Geodesy Photogrammetry and Cartography, Vol. 27, No. 6, 2009, pp. 659-667.

17. Kleijer, F., Odijk, D., and Verbree, E., "Prediction of GNSS Availability and Accuracy in Urban Environments - Case Study Schiphol Airport," Chapter 23 of Location Based Services and TeleCartography II, G. G.a. K. Rehrl, Editor, Springer-Verlag, Berlin Heidelberg, 2009, pp. 387-406.

18. Gang-jun, L., et al., "Characterisation of Current and Future GNSS Performance in Urban Canyons Using a High Quality 3-D Urban Model of Melbourne, Australia," Journal of Applied Geodesy, 2009, p. 15.
19. Ji, S., et al., "Potential Benefits of GPS/GLONASS/ GALILEO Integration in an Urban Canyon - Hong Kong," The Journal of Navigation, Vol. 63, No. 4, 2010, pp. 681-693.

20. Costa, E., "Simulation of the Effects of Different Urban Environments on GPS Performance Using Digital Elevation Models and Building Databases," IEEE Transactions on Intelligent Transportation Systems, Vol. 12, No. 3, 2011, pp. 819-829.

21. Peyret, F., Bétaille, D., and Mougel F., "Non-Line-OfSight GNSS Signal Detection Using an On-Board 3D Model of Buildings," $11^{\text {th }}$ International Conference on ITS Telecommunications (ITST), 2011.

22. Obst, M., Bauer, S., and Wanielik, G., "Urban Multipath Detection and Mitigation with Dynamic 3D Maps for Reliable Land Vehicle Localization," Position Location and Navigation Symposium (PLANS), 2012.

23. Bourdeau, A., Sahmoudi, M., and Tourneret, J.-Y., "Tight Integration of GNSS and a 3D City Model for Robust Positioning in Urban Canyons," Proceedings of the $25^{\text {th }}$ International Technical meeting of the Satellite Division of The Institute of Navigation (ION GNSS 2012), Nashville, TN, September 2012, pp. 1263-1269.

24. Piñana-Díaz, C., et al., "A Two-Layers Based Approach of an Enhanced-Mapfor Urban Positioning Support," Sensors, Vol. 12, No. 11, 2012, pp. 14508-14524.

25. Peyraud, S., et al., "About Non-Line-Of-Sight Satellite Detection and Exclusion in a 3D Map-Aided Localization Algorithm," Sensors, Vol. 13, No. 1, 2013, pp. 829-847.

26. Cappelle, C., et al., "Virtual 3D City Model for Navigation in Urban Areas," Journal of Intelligent and Robotic Systems, Vol. 66, No. 3, 2012, pp. 377-399. DOI: $10.1007 / \mathrm{s} 10846-011-9594-0$.

27. Tiberius, C., and Verbree, E., "GNSS Positioning Accuracy and Availability within Location Based Services: The Advantages of Combined GPS-Galileo Positioning," NaviTec, 2004.

28. Wang, L., Groves, P., and Ziebart, M., "GNSS Shadow Matching Using A 3D Model of London," European Navigation Conference, Grange Tower Bridge, London, 2011.

29. Groves, P. D., Wang, L., and Ziebart, M., "Shadow Matching Improved GNSS Accuracy in Urban Canyons," GPS World, Vol. 23, No. 2, 2012a, pp. 14-29.

30. Jiang, Z., and Groves, P. D., "GNSS NLOS and Multipath Error Mitigation using Advanced MultiConstellation Consistency Checking with Height Aiding," Proceedings of the $25^{\text {th }}$ International Technical Meeting of the Satellite Division of The Institute of Navigation (ION GNSS 2012), Nashville, TN, September 2012, pp. 79-88.

31. Walker, R., and Kubik, K., "Numerical Modelling of GPS Signal Propagation," Proceedings of the 9th International Technical Meeting of the Satellite Division of The Institute of Navigation (ION GPS 1996), Kansas City, MO, pp. 709-717.

32. Groves, P. D., Jiang, Z., Wang, L., and Ziebart, M. K.., "Intelligent Urban Positioning using MultiConstellation GNSS with 3D Mapping and NLOS Signal Detection," Proceedings of the $25^{\text {th }}$ International Technical Meeting of the Satellite Division of The Institute of Navigation (ION GNSS 2012), Nashville, TN, September 2012, pp. 458-472. 